\title{
LAGUERRE-TYPE BELL POLYNOMIALS
}

\author{
P. NATALINI AND P. E. RICCI
}

Received 14 October 2005; Revised 4 May 2006; Accepted 9 May 2006

We develop an extension of the classical Bell polynomials introducing the Laguerre-type version of this well-known mathematical tool. The Laguerre-type Bell polynomials are useful in order to compute the $n$th Laguerre-type derivatives of a composite function. Incidentally, we generalize a result considered by L. Carlitz in order to obtain explicit relationships between Bessel functions and generalized hypergeometric functions.

Copyright (c) 2006 Hindawi Publishing Corporation. All rights reserved.

\section{Introduction}

The Bell polynomials [1] appear in different frameworks. They are often used in combinatorial analysis [20], and even in statistics [14], although without explicit references. Moreover these polynomials have been applied even in many other contexts, such as the Blissard problem (see [20, page 46]), the representation of Lucas polynomials of the first and second kinds $[4,9]$, the representation formulas of Newton sum rules for polynomials' zeros [12, 13], the recurrence relations for a class of Freud-type polynomials [3], the representation of symmetric functions of a countable set of numbers, generalizing the classical algebraic Newton-Girard formulas [15]. Consequently they were also used [6] in order to find reduction formulas for the orthogonal invariants of a strictly positive compact operator, deriving in a simple way the so-called Robert formulas [21].

Some generalized forms of Bell polynomials already appeared in literature (see, e.g., $[11,17,19])$. A generalization of the Bell polynomials suitable for the differentiation of multivariable composite functions can also be found in [18]. Lastly, in [2], the socalled multidimensional Bell polynomials of higher order were introduced, which are suitable for representing the derivative of a composite function of several (say $m$ ) variables $f\left(\varphi^{(1)}(t), \varphi^{(2)}(t), \ldots, \varphi^{(m)}(t)\right)$, where $\varphi^{(i)}(t)=\phi^{(i, 1)}\left(\phi^{(i, 2)}\left(\cdots \phi^{\left(i, r_{i}\right)}(t)\right)\right),(i=1,2, \ldots, m)$.

In this article we find explicit representation formulas for the $n$th Laguerre-type derivatives of a composite function. The case of the first Laguerre derivative $D x D, D:=$ $d / d x$ is essentially related to an article by Carlitz [5], originated by a preceding paper by Lardner [16] in which the powers $(D x D)^{n}$ of this derivative appear. 


\section{Recalling the Bell polynomials}

We recall that the Bell polynomials are a classical mathematical tool for representing the $n t h$ derivative of a composite function. In fact by considering the composite function $\Phi(t):=f(g(t))$ of functions $x=g(t)$ and $y=f(x)$ defined in suitable intervals of the real axis and $n$ times differentiable with respect to the relevant independent variables and by using the following notations:

$$
\begin{gathered}
\Phi_{h}:=D_{t}^{h} \Phi(t), \quad f_{h}:=\left.D_{x}^{h} f(x)\right|_{x=g(t)}, \quad g_{h}:=D_{t}^{h} g(t), \\
\left([f, g]_{n}\right):=\left(f_{1}, g_{1} ; f_{2}, g_{2} ; \ldots ; f_{n}, g_{n}\right),
\end{gathered}
$$

they are defined as follows:

$$
Y_{n}\left([f, g]_{n}\right):=\Phi_{n}
$$

For example one has

$$
\begin{gathered}
Y_{1}\left([f, g]_{1}\right)=f_{1} g_{1}, \\
Y_{2}\left([f, g]_{2}\right)=f_{1} g_{2}+f_{2} g_{1}^{2}, \\
Y_{3}\left([f, g]_{3}\right)=f_{1} g_{3}+f_{2}\left(3 g_{2} g_{1}\right)+f_{3} g_{1}^{3} .
\end{gathered}
$$

Further examples can be found in [20, page 49].

Inductively, we can write

$$
Y_{n}\left([f, g]_{n}\right)=\sum_{k=1}^{n} A_{n, k}\left(g_{1}, g_{2}, \ldots, g_{n}\right) f_{k}
$$

where the coefficient $A_{n, k}$, for any $k=1, \ldots, n$, is a polynomial in $g_{1}, g_{2}, \ldots, g_{n}$, homogeneous of degree $k$ and isobaric of weight $n$ (i.e., it is a linear combination of monomials $g_{1}^{k_{1}} g_{2}^{k_{2}} \cdots g_{n}^{k_{n}}$ whose weight is constantly given by $\left.k_{1}+2 k_{2}+\cdots+n k_{n}=n\right)$.

For them the following result holds true.

Proposition 2.1. The Bell polynomials satisfy the recurrence relation

$$
\begin{gathered}
Y_{0}\left([f, g]_{0}\right):=f_{1}, \\
Y_{n+1}\left([f, g]_{n+1}\right)=\sum_{k=0}^{n}\left(\begin{array}{l}
n \\
k
\end{array}\right) Y_{n-k}\left(\left[f_{1}, g\right]_{n-k}\right) g_{k+1},
\end{gathered}
$$


where

$$
\left(\left[f_{1}, g\right]_{n-k}\right):=\left(f_{2}, g_{1} ; f_{3}, g_{2} ; \ldots ; f_{n-k+1}, g_{n-k}\right)
$$

An explicit expression for the Bell polynomials is also given by the Faà di Bruno formula [10]:

$$
\Phi_{n}=Y_{n}\left([f, g]_{n}\right)=\sum_{\pi(n)} \frac{n !}{j_{1} ! j_{2} ! \cdots j_{n} !} f_{j}\left[\frac{g_{1}}{1 !}\right]^{j_{1}}\left[\frac{g_{2}}{2 !}\right]^{j_{2}} \cdots\left[\frac{g_{n}}{n !}\right]^{j_{n}},
$$

where the sum runs over all partitions $\pi(n)$ of the integer $n$, that is, $n=j_{1}+2 j_{2}+\cdots+$ $n j_{n}$. In (2.7) $j_{h}$ denotes the number of parts of size $h$, and $j=j_{1}+j_{2}+\cdots+j_{n}$ denotes the number of parts of the considered partition. A proof of the Faà di Bruno formula can be found in [20]. In [22] the proof is based on the umbral calculus (see [23] and the references therein).

\section{Laguerre-type derivatives}

The Laguerre-type derivatives were introduced in $[7,8]$ in connection with a differential isomorphism denoted by the symbol $\mathscr{T}:=\mathscr{T}_{x}$, acting onto the space $\mathscr{A}:=\mathscr{A}_{x}$ of analytic functions of the $x$ variable by means of the correspondence

$$
D:=\frac{d}{d x} \longrightarrow \hat{D}_{L}:=D x D ; \quad x \cdot \longrightarrow \hat{D}_{x}^{-1},
$$

where

$$
\begin{gathered}
\hat{D}_{x}^{-1} f(x):=\int_{0}^{x} f(\xi) d \xi \\
\hat{D}_{x}^{-n} f(x):=\frac{1}{(n-1) !} \int_{0}^{x}(x-\xi)^{n-1} f(\xi) d \xi
\end{gathered}
$$

so that

$$
\mathscr{T}_{x}\left(x^{n}\right)=\hat{D}_{x}^{-n}(1):=\frac{1}{(n-1) !} \int_{0}^{x}(x-\xi)^{n-1} d \xi=\frac{x^{n}}{n !}
$$

According to this isomorphism, the exponential operator $e^{x}$ is transformed into the first Laguerre-type exponential $e_{1}(x):=\sum_{k=0}^{\infty} x^{k} /(k !)^{2}$ which is an eigenfunction of the Laguerre derivative operator $D_{L}:=D x D$. We have, in fact,

$$
\begin{gathered}
\mathscr{T}_{x}\left(e^{x}\right)=\sum_{k=0}^{\infty} \frac{\mathcal{T}_{x}\left(x^{k}\right)}{k !}=\sum_{k=0}^{\infty} \frac{x^{k}}{(k !)^{2}}=e_{1}(x), \\
\hat{D}_{L} e_{1}(a x)=a e_{1}(a x), \quad \forall a \in \mathbb{C} .
\end{gathered}
$$


This result can be generalized by considering the $r$ Laguerre-type exponential $e_{r}(x):=$ $\sum_{k=0}^{\infty} x^{k} /(k !)^{r+1}$, the $r$ th Laguerre-type derivative operator $D_{r L}:=D x D x D \cdots D x D$ (containing $r+1$ ordinary derivatives), and the iterated isomorphism $\mathscr{T}^{r}$, since

$$
\begin{gathered}
\mathscr{T}_{x}^{r}\left(e^{x}\right)=\sum_{k=0}^{\infty} \frac{\mathcal{T}_{x}\left(x^{k}\right)}{(k !)^{r}}=\sum_{k=0}^{\infty} \frac{x^{k}}{(k !)^{r+1}}=e_{r}(x), \\
\hat{D}_{r L} e_{r}(a x)=a e_{r}(a x), \quad \forall a \in \mathbb{C} .
\end{gathered}
$$

Remark 3.1. The above results show that, for every positive integer $r$, we can define a Laguerre-type exponential function $e_{r}(x)$, satisfying an eigenfunction property, which is an analog of the elementary property of the exponential. This function reduces to the exponential function when $r=0$, so that we can put by definition

$$
e_{0}(x):=e^{x}, \quad \hat{D}_{0 L}:=D \text {. }
$$

Obviously, $\hat{D}_{1 L}:=\hat{D}_{L}$.

For this reason we will refer to such functions as $L$-exponential functions, or shortly $L$-exponentials.

\section{Laguerre-type Bell polynomials}

The problem of constructing Bell polynomials can be extended in the natural way to the case of the Laguerre-type derivatives.

To this aim, by using notations in (2.1), we introduce the following definition.

Definition 4.1. The $n$th Laguerre-type Bell polynomial, denoted by ${ }_{r L} Y_{n}\left(x ;[f, g]_{n}\right)$, represents the $n$th $r$ Laguerre-type derivative of the composite function $f(g(t))$.

We will show that ${ }_{r L} Y_{n}$ can be expressed as a polynomial in the independent variable $x$, depending on $f_{1}, g_{1} ; f_{2}, g_{2} ; \ldots ; f_{n}, g_{n}$ in terms of the classical Bell polynomials.

We start noting that, according to a general result due to Viskov [24], the Laguerre derivative satisfy

$$
\left(D_{L}\right)^{n}=(D x D)^{n}=D^{n} x^{n} D^{n},
$$

and furthermore, for any order $r$, it turns out that

$$
\left(D_{r L}\right)^{n}=(D x D x \cdots D x D)^{n}=D^{n} x^{n} D^{n} x^{n} \cdots D^{n} x^{n} D^{n} .
$$

According to the above equations, the proof of Carlitz [5] can be reduced to a simple application of the Leibnitz rule, since

$$
\begin{aligned}
(D x D)^{n} & =D^{n}\left(x^{n} D^{n}\right)=\sum_{k=0}^{n}\left(\begin{array}{l}
n \\
k
\end{array}\right) D^{n-k} x^{n} D^{n+k} \\
& =\sum_{k=0}^{n}\left[\left(\begin{array}{l}
n \\
k
\end{array}\right)\right]^{2}(n-k) ! x^{k} D^{n+k}=\sum_{k=0}^{n} \frac{n !}{k !}\left(\begin{array}{l}
n \\
k
\end{array}\right) x^{k} D^{n+k} .
\end{aligned}
$$


Therefore, the following representation formula for the Laguerre-type Bell polynomials, denoted by ${ }_{L} Y_{n}$, holds true.

THeOREM 4.2. The ${ }_{L} Y_{n}$ polynomials are expressed in terms of the ordinary Bell polynomials according to the equation

$$
{ }_{L} Y_{n}\left(x ;[f, g]_{n}\right)=\sum_{k=0}^{n} \frac{n !}{k !}\left(\begin{array}{l}
n \\
k
\end{array}\right) x^{k} Y_{n+k}\left([f, g]_{n+k}\right) .
$$

The above results can be easily generalized, since

$$
\begin{aligned}
\left(D_{2 L}\right)^{n} & =(D x D x D)^{n}=D^{n} x^{n}\left(D^{n} x^{n} D^{n}\right) \\
& =\sum_{k_{1}=0}^{n} \sum_{k_{2}=0}^{n} \frac{n !}{k_{1} !} \frac{\left(n+k_{1}\right) !}{\left(k_{1}+k_{2}\right) !}\left(\begin{array}{c}
n \\
k_{1}
\end{array}\right)\left(\begin{array}{c}
n \\
k_{2}
\end{array}\right) x^{k_{1}+k_{2}} D^{n+k_{1}+k_{2}} .
\end{aligned}
$$

\section{The general case}

The following result follows by induction.

Theorem 5.1. The powers of the rth Laguerre-type derivative operator $D_{r L}:=D x D x D \cdots$ $D x D$ (containing $r+1$ ordinary derivatives) can be expanded in the form

$$
\begin{aligned}
\left(D_{r L}\right)^{n}= & (D x D x \cdots D x D)^{n}=D^{n} x^{n} D^{n} x^{n} \cdots D^{n} x^{n} D^{n} \\
= & \sum_{k_{1}=0}^{n} \sum_{k_{2}=0}^{n} \cdots \sum_{k_{r}=0}^{n} \frac{n !}{k_{1} !} \frac{\left(n+k_{1}\right) !}{\left(k_{1}+k_{2}\right) !} \cdots \frac{\left(n+k_{1}+k_{2}+\cdots+k_{r-1}\right) !}{\left(k_{1}+k_{2}+\cdots+k_{r}\right) !} \\
& \times\left(\begin{array}{c}
n \\
k_{1}
\end{array}\right)\left(\begin{array}{c}
n \\
k_{2}
\end{array}\right) \cdots\left(\begin{array}{c}
n \\
k_{r}
\end{array}\right) x^{k_{1}+k_{2}+\cdots+k_{r}} D^{n+k_{1}+k_{2}+\cdots+k_{r}} .
\end{aligned}
$$

Therefore, for the $r$ th Laguerre-type Bell polynomials denoted by ${ }_{r L} Y_{n}$, the following result holds true.

THeOREM 5.2. The ${ }_{r L} Y_{n}$ polynomials are expressed in terms of the ordinary Bell polynomials according to the equation

$$
\begin{aligned}
{ }_{r L} Y_{n}\left(x ;[f, g]_{n}\right)= & \sum_{k_{1}=0}^{n} \sum_{k_{2}=0}^{n} \cdots \sum_{k_{r}=0}^{n} \frac{n !}{k_{1} !} \frac{\left(n+k_{1}\right) !}{\left(k_{1}+k_{2}\right) !} \cdots \frac{\left(n+k_{1}+k_{2}+\cdots+k_{r-1}\right) !}{\left(k_{1}+k_{2}+\cdots+k_{r}\right) !} \\
& \times\left(\begin{array}{c}
n \\
k_{1}
\end{array}\right)\left(\begin{array}{c}
n \\
k_{2}
\end{array}\right) \cdots\left(\begin{array}{c}
n \\
k_{r}
\end{array}\right) x^{k_{1}+k_{2}+\cdots+k_{r}} Y_{n+k_{1}+k_{2}+\cdots+k_{r}}\left([f, g]_{n+k_{1}+k_{2}+\cdots+k_{r}}\right) .
\end{aligned}
$$




\section{Acknowledgments}

This paper was concluded in the framework of the Italian National Group for Scientific Computation (GNCS). Useful discussions with Professor Dr. Y. Ben Cheikh are gratefully recognized.

\section{References}

[1] E. T. Bell, Exponential polynomials, Annals of Mathematics. Second Series 35 (1934), no. 2, 258 277.

[2] A. Bernardini, P. Natalini, and P. E. Ricci, Multidimensional Bell polynomials of higher order, Computers \& Mathematics with Applications 50 (2005), no. 10-12, 1697-1708.

[3] A. Bernardini and P. E. Ricci, Bell polynomials and differential equations of Freud-type polynomials, Mathematical and Computer Modelling 36 (2002), no. 9-10, 1115-1119.

[4] M. Bruschi and P. E. Ricci, I polinomi di Lucas e di Tchebycheff in più variabili [Lucas and Čebyšev polynomials in several variables], Rendiconti di Matematica. Serie VI 13 (1980), no. 4, 507-529 (1981).

[5] L. Carlitz, Some reduction formulas for generalized hypergeometric functions, SIAM Journal on Mathematical Analysis 1 (1970), 243-245.

[6] C. Cassisa and P. E. Ricci, Orthogonal invariants and the Bell polynomials, Rendiconti di Matematica e delle sue Applicazioni. Serie VII 20 (2000), 293-303.

[7] G. Dattoli and P. E. Ricci, Laguerre-type exponentials, and the relevant L-circular and L-hyperbolic functions, Georgian Mathematical Journal 10 (2003), no. 3, 481-494.

[8] G. Dattoli and A. Torre, Operatorial methods and two variable Laguerre polynomials, Atti della Accademia delle Scienze di Torino. Classe di Scienze Fisiche, Matematiche e Naturali 132 (1998), 3-9.

[9] A. Di Cave and P. E. Ricci, Sui polinomi di Bell ed i numeri di Fibonacci e di Bernoulli [On Bell polynomials and Fibonacci and Bernoulli numbers], Le Matematiche 35 (1980), no. 1-2, 84-95.

[10] F. Faà di Bruno, Théorie des formes binaires, Brero, Turin, 1876.

[11] D. Fujiwara, Generalized Bell polynomials, Sūgaku (Mathematics) 42 (1990), no. 1, 89-90.

[12] T. Isoni, P. Natalini, and P. E. Ricci, Symbolic computation of Newton sum rules for the zeros of orthogonal polynomials, Advanced Special Functions and Integration Methods (Melfi, 2000), Proc. Melfi Sch. Adv. Top. Math. Phys., vol. 2, Aracne, Rome, 2001, pp. 97-112.

[13] Symbolic computation of Newton sum rules for the zeros of polynomial eigenfunctions of linear differential operators, Numerical Algorithms 28 (2001), no. 1-4, 215-227, special volume in memory of W. Gross.

[14] M. G. Kendall and A. Stuart, The Advanced Theory of Statistics, Griffin, London, 1958.

[15] A. Kurosh, Cours d'Algèbre Supérieure, Éditions Mir, Moscow, 1971.

[16] T. J. Lardner, Relations between ${ }_{0} F_{3}$ and Bessel functions, SIAM Review 11 (1969), 69-72.

[17] P. Natalini and P. E. Ricci, An extension of the Bell polynomials, Computers \& Mathematics with Applications 47 (2004), no. 4-5, 719-725.

[18] S. Noschese and P. E. Ricci, Differentiation of multivariable composite functions and Bell polynomials, Journal of Computational Analysis and Applications 5 (2003), no. 3, 333-340.

[19] P. N. Rai and S. N. Singh, Generalization of Bell polynomials and related operational formula, Vijnana Parishad Anusandhan Patrika 25 (1982), no. 3, 251-258 (Hindi).

[20] J. Riordan, An Introduction to Combinatorial Analysis, Wiley Publications in Mathematical Statistics, John Wiley \& Sons, New York, 1958.

[21] D. Robert, Invariants orthogonaux pour certaines classes d'opérateurs, Journal de Mathématiques Pures et Appliquées. Neuvième Série 52 (1973), 81-114.

[22] S. M. Roman, The formula of Fad di Bruno, The American Mathematical Monthly 87 (1980), no. $10,805-809$. 
[23] S. M. Roman and G.-C. Rota, The umbral calculus, Advances in Mathematics 27 (1978), no. 2, 95-188.

[24] O. V. Viskov, A commutative-like noncommutative identity, Acta Scientiarum Mathematicarum (Szeged) 59 (1994), no. 3-4, 585-590.

P. Natalini: Dipartimento di Matematica, Università di Roma Tre, Largo S. Leonardo Murialdo 1, 00146 Roma, Italy

E-mail address: natalini@mat.uniroma3.it

P. E. Ricci: Dipartimento di Matematica, Università delgi Studi di Roma "La Sapienza,"

P. le Aldo Moro 2, 00185 Roma, Italy

E-mail address: paoloemilio.ricci@uniroma1.it 


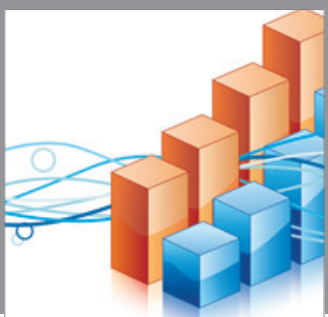

Advances in

Operations Research

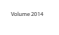

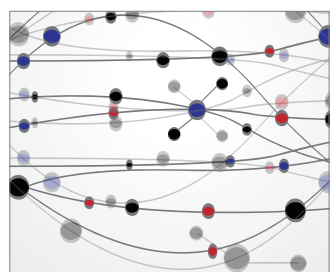

\section{The Scientific} World Journal
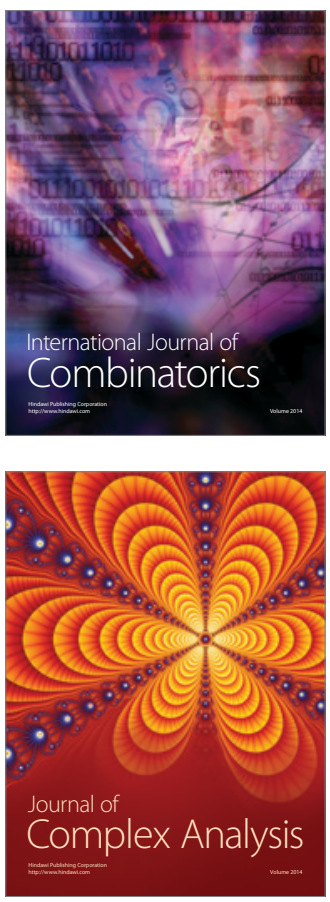

International Journal of

Mathematics and

Mathematical

Sciences
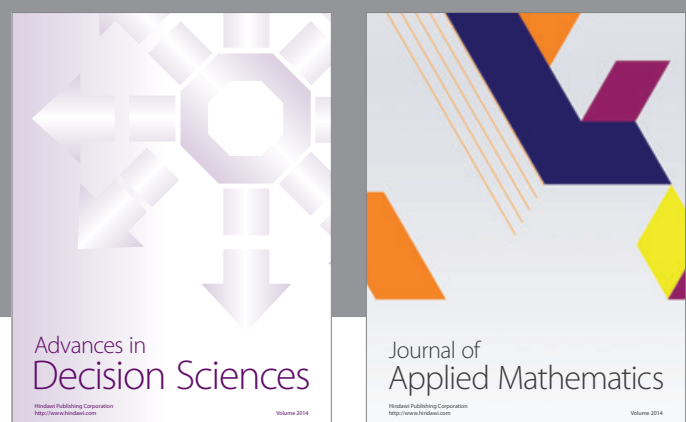

Journal of

Applied Mathematics
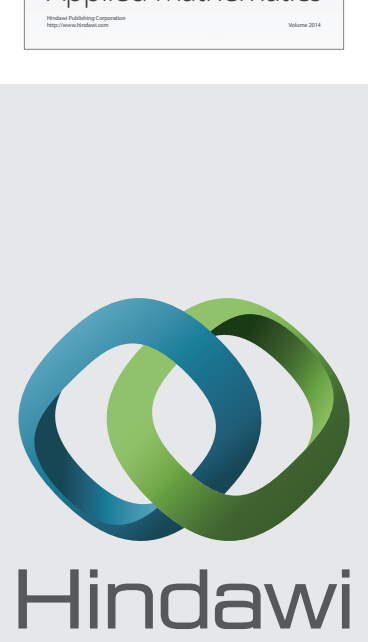

Submit your manuscripts at http://www.hindawi.com
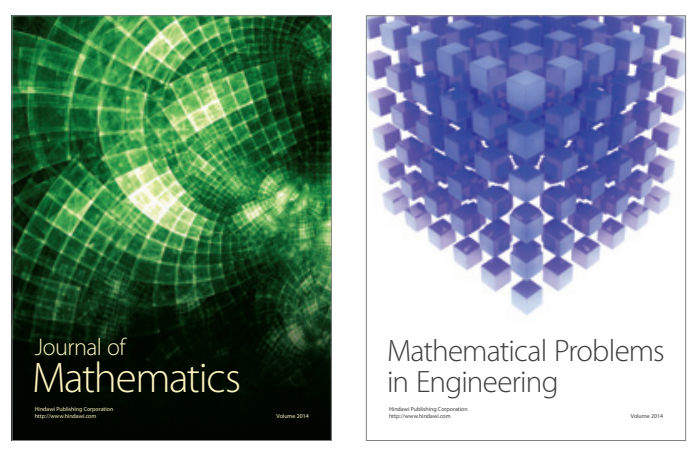

Mathematical Problems in Engineering
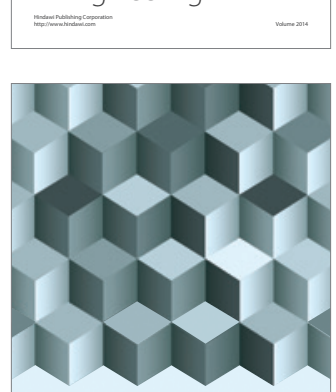

Journal of

Function Spaces
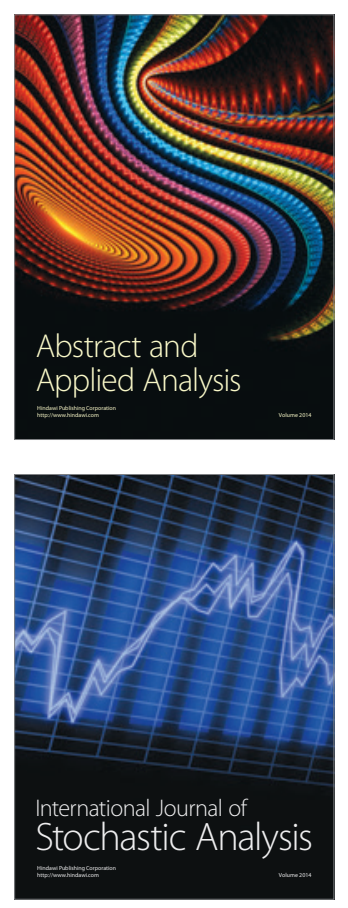

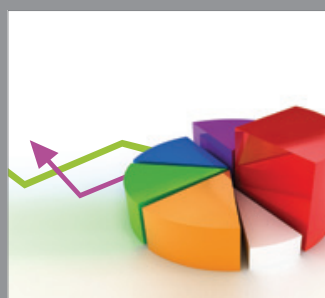

ournal of

Probability and Statistics

Promensencen
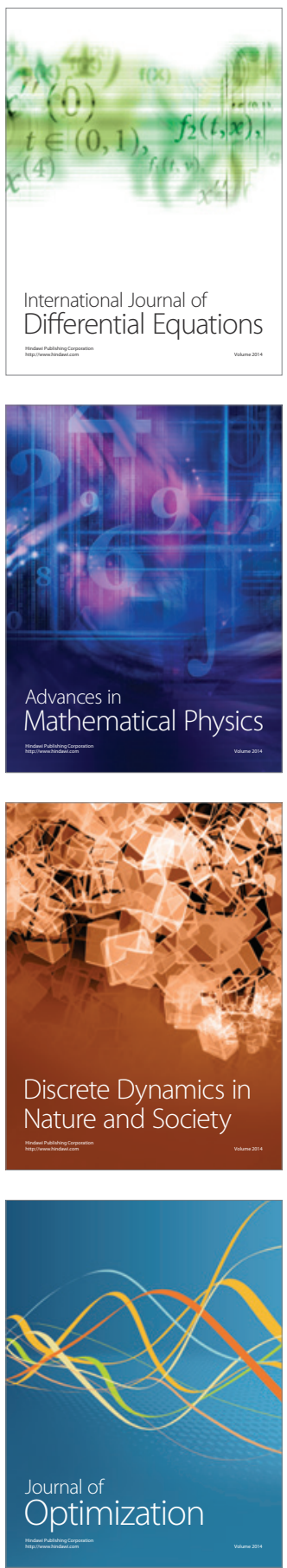